\title{
Umkehrbare Entmischung der Kernkolloide nach Vitalfärbung und Plasmolyse
}

\author{
Von \\ Otto Bank \\ Aus dem Institut für allgemeine Biologie der Masarykuniversität \\ in Bruinn
}

\section{I}

STRUGGER (1933) konnte einwandfrei zeigen, daß die Kernstrukturen reversible Entmischungsformen des Karyotins sind. Bemerkenswert ist, daß saure Vitalfarbstoffe nach demselben Autor nur den Kernsaft färben, nicht aber die Strukturen, basische dagegen das Karyotin. BECKER und BECKerowa (1933) konnten mit basischen Farbstoffen, bei Benützung des elektrischen Stromes, Chromatin und Nukleolen umkehrbar färben. Gleichzeitig weisen die beiden Autoren darauf hin, daß die bisher durchgeführten Vitalfärbungen des pflanzlichen Zellkernes (GICKLHORN 1927; ALBACH 1929; BANK 1933) mit sauren sowie mit basischen Farbstoffen (BANK 1933) nur an sehr stark pathologisch veränderten Kernen gelungen ist, wenn man nach dem Untersuchungen verschiedener Autoren (Martens 1927; SharP 1929; TELEŻYŃSKI 1930; NEBEL 1932, u. a. zitiert nach BECKER u. BECKERowa) die netzartige Struktur der Allium-Kerne als die normale betrachtet. Nun scheint mir (BANK 1936) durch die Arbeit StRUgGers (1933) das Problem der Normalstruktur des Kernes in viel beweglicherem und einschlägigerem Sinne gelöst zu sein, demzufolge die jeweilige Struktur Funktion ist gewisser Lebensbedingungen ( $\mathrm{pH}$, Salzkonzentration). Daher war es auf Grund dieser Erkenntnis notwendig, experimentell zu überprüfen, inwieweit namentlich der Einwand BECKER und BECKERowAs stichhältig ist, da $\beta$ die von mir mit basischen Farbstoffen mit Hilfe der Plasmolyse gefärbten Kerne in ihrem Chromatinanteil verändert sind. Diese Überprüfung schien umso interessanter zu werden, als die mit basischen Farbstoffen bei gleichzeitiger Plasmolyse gefärbten Kerne in ihrer Färbung reversibel sind (BANK 1935). Es bleibt dabei unbestritten, daß durch die zur Erzielung der Vitalfärbung mit der von mir angewandten Methode neue, von den normalen verschiedene Gleichgewichte im behandelten lebenden Systeme (BertalaNFFY) eintreten können. Fraglich bleibt jedoch, ob diese Veränderungen gerade den Chromatinanteil des Kernes betreffen müssen, was BECKer u. BECKERowa für d̃ie Erklärung der von mir erhaltenen Diffusfär- 
bung des Kernes, mit basischen Farbstoffen erzielt, für wichtig zu halten scheinen. Es könnte im Sinne des vorher Gesagten das gesamte Kernsystem, vielleicht auch das Zellsystem in einer typischen Weise verändert sein,was die Diffusfärbung bedingte. Und so wird in der vorliegenden Mitteilung der Nachweis zu erbringen versucht, dafs das Chromatin in den von mir vitalgefärbten Kernen tatsächlich in reversibel strukturierbarer Form im Kerne vorhanden ist, daß somit die diffuse Vitalfärbung der Kerne mit basischen Farbstoffen in plasmolysierten Zellen auf einer Veränderung der Gleichgewichte im lebenden System beruht und nicht auf dem Mangel eines bestimmten Stoffes, lies: des Chromatins.

\section{II}

Versuchsmaterial: Zellen der inneren Epidermis von Allium cepa.

Nach langen Vorversuchen gelang es, dieses sichere Behandlungsrezept zu finden das analog ist jenem, das angewandt worden ist um in der Zellsaftvakuole Entmischungen zu erzielen: die Epidermen werden für 10-15 Minuten in ein Gemisch von $1 \mathrm{~mol} \mathrm{KCl}$ und $0.1 \%$ Methylviolett $(1: 1)$ eingelegt. Nach dieser Zeit sind die Kerne in den plasmolysierten Zellen diffus blau gefärbt. Auf dem Deckglase, bei mikroskopischer Beobachtung, wird 1 mol KSCN zugesetzt,

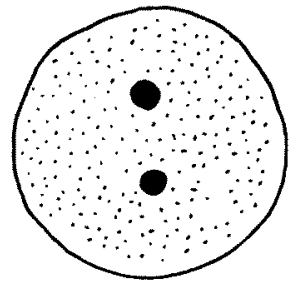

Fig. 1. Ein körnig entmischter Zellkern nach diffuser Vitalfärbung. Körner und Nukleolen sind mit Farbstoff besetzt, die Zwischensub$\operatorname{stan} z$ ist farblos. worauf, nach einer Wirkungsdauer von einigen Minuten, die Kerne violett werden und die umkehrbare Entmischung von Nukleolen und Körnern eintritt.

\section{III}

(A) Bei weiterer Beobachtung der entmischten Kerne sehen wir, daß Körner (Granula) und Nukleolen wieder verschwinden, daß die Kerne glasig werden und schließlich, bei gleichzeitiger, sehr feinkörniger Entmischung platzen. Das ist der erste (1) Modus der umkehrbaren Entmischung der mit dem basischen Farbstoffe diffus gefärbten Kerne. Wir besitzen noch einen zweiten (2) ohne notwendig tötlichen Ausgang: sofort nach dem Erscheinen der Nukleolen und Körner im vorhin diffus gefärbten Kerne, übertragen wir die Zellen in reine 1 mol KCl-Lösung. Nach einiger Zeit sind die Kerne wieder diffus blau geworden und können durch Zugabe von 1 mol KSCN-Lösung in wiederum reversibler Weise körnig entmischt werden. Durch rechtzeitigen Wechsel der beiden Lösungen ( $\mathrm{KCl} \mathrm{u}$. $\mathrm{KSCN}$ ) gelingt es, diese reversible Entmischung zu wiederholen. In mehreren Fällen 
konnte ich das dreimal erzielen. Weiter konnte diese Wiederholung nicht getrieben werden, weil die Zellen doch leiden und schließlich zugrunde gehen, und zwar aus der entmischten Form auf die unter Modus (1) beschriebene Weise, aus der diffusen Form (ohne Körner u. Nukleolen) direkt durch Platzen bei gleichzeitiger, uns schon bekannter, sehr feinkörniger Entmischung.

Außerdem können die Entmischungskörner durch verstärkte Plasmolyse zum Verschwinden gebracht, durch Deplasmolyse wieder erzeugt werden. Diese Behandlung ist jedoch ziemlich schwierig.

(B) Es ist somit sicher, daß der mit einem basischen Farbstoffe in der plasmolysierten Zelle diffus gefärbte Kerninhalt reversibel zu Körnern entmischbar ist. Trotzdem normalerweise bei der von uns beschriebenen Entmischungsmethode mit den Körnern auch die Nukleolen erscheinen, könnten Zweifel auftauchen, ob denn die entstandenen Körner auch tatsächlich aus chromatischer Substanz, wenigstens dem Hauptanteile nach, bestehen. Diese Zweifel zu beheben, scheinen wir in der Methode Struggers (1930), der reversiblen Retikulumdarstellung im Zellkern, ein Mittel zu besitzen. Bekanntlich gelingt es, in bestimmt konzentrierten $\mathrm{KNO}_{3}$-Lösungen das Kernretikulum im Alliumkern darzustellen oder es unsichtbar werden zu lassen. Dasselbe gelingt auch in gleichkonzentrierten KCl-Lösungen, die für unsere Zwecke von besonderer Wichtigkeit sind. So können wir in Konzentrationen von etwa 0.05 mol Kerne ohne Retikulum, jedoch mit sichtbaren Nukleolen darstellen, in Konzentrationen von etwa $0.17 \mathrm{~mol}$ erhalten wir Kerne mit Retikulum und Nukleolen, in Konzentrationen von $0.4 \mathrm{~mol}$ erhalten wir ganz homogene Kerne ohne Retikulum und ohne Nukleolen. Es ist also anscheinend die Möglichkeit gegeben, in vorher ganz bestimmt strukturierten und hierauf gefärbten Kernen (1) die Lokalisation des Farbstoffes festzustellen und (2) in gefärbten Kernen mit vorhandenem Retikulum die Kernkolloide $z$ entmischen versuchen, wobei das etwaige Mißlingen der Entmischung in solchen Kernen mit einer gehörigen Vorsicht als Beweis dafür gelten kann, daß die unter (A) beschriebenen Entmischungskörner zu großem Teil aus chromatischer Substanz bestehen.

Die praktische Durchführung dieser Möglichkeiten ist leider schwieriger und nicht so eindeutig wie man im Vorhinein anzunehmen geneigt wäre. Denn die Applikation der Farbstofflösung im Gemisch mit niedrig konzentrierten Salzlösungen, dazu noch auf bereits behandelte Zellen, wirkt sehr giftig, außardem wirkt eine $0.17 \mathrm{~mol}$ Salzlösung im Gemisch mit $0.1 \%$ Methylviolett anders als die reine Salzlösung. Wir erhalten somit nur ,launische“ Resultate, Resultate mit großer Variationsbreite: aus gleichem Gemisch (etwa $0.17 \mathrm{~mol}$ 
$\mathrm{KCl}+$ Methylviolett) erhalten wir nach derselben Wirkungsdauer einmal diffus gefärbte, das anderemal Kerne mit gefärbter körniger Struktur. Die Entmischung mit gleichmolarer KSCN-Lösung ist nicht so sicher zu erhalten wie bei der bei (II) beschriebenen Behandlung. Sicher jedoch ist soviel, daß in Kernen mit körniger Struktur auf keinen Fall eine zweite Entmischung stattfindet, während sie in diffus gefärbten Kernen zu erzielen ist. Wichtig aber ist, daß die körnige gefärbte Struktur der Kerne durch Plasmolyse zum Verschwinden gebracht werden kann. Weiter muß erwähnt werden, daß in den diffus gefärbten Kernen aus dem Gemisch $0.05 \mathrm{~mol} \mathrm{KCl}+0.1 \%$ Methylviolett $(1: 1)$ mit $0.05 \mathrm{~mol}$ KSCN keine Entmischung erzielt werden konnte, so daß es den Anschein hat, als wäre die Entmischung nach unserer Methode erst von einer bestimmten höheren Konzentration ab zu erzielen.

Obwohl, wie aus dem Berichteten ersichtlich, die gestellte Aufgabe nicht klar gelöst werden konnte, können wir schon jetzt mit einigem guten Recht annehmen, daß die Entmischungskörner wenigstens teilweise aus jener Substanz bestehen (dem Karyotin) die bei Struggers Methode das Kernretikulum bildet und die von Becker u. BECKERowa in Anlehnung an die schon genannten Autoren als Chromatin bezeichnet wird.

(C) Diese Annahme wird durch die nun zu berichtenden Versuchsergebnisse bestätigt. Bevor wir jedoch darüber berichten müssen wir weiter ausholen: es ist selbstverständlich, daß das unter II angegebene und bis jetzt angewandte Behandlungsrezept der Zwiebelepidermen auf seine Berechtigung näher untersucht wurde. Daher wurde die Wirkung anderer in ihrer kernfärberischen Wirkung ähnlicher Farbstoffe wie Gentiana und Kristallviolett, Prune pure, Malachitgrün, Neutralrot außərdem die Wirkung anderer dem Farbstoffe beigemischter Salze untersucht. Es waren dies: $\mathrm{NH}_{4} \mathrm{NO}_{3}$, $\mathrm{KNO}_{3}, \mathrm{NaNO}_{3}, \mathrm{Ca}\left(\mathrm{NO}_{3}\right)_{2} ;\left(\mathrm{NH}_{4}\right)_{2} \mathrm{SO}_{4}, \mathrm{Na}_{2} \mathrm{SO}_{4}, \mathrm{~K}_{2} \mathrm{SO}_{4} ; \mathrm{NH}_{4} \mathrm{Cl}$, $\mathrm{LiCl}, \mathrm{NaCl}$. Als Entmischungssalz wurde außerdem noch $\mathrm{NH}_{4} \mathrm{SCN}$ benützt.

Bei Verwendung aller Chloride als Beimischungssalze zu Methylviolett erhalten wir bei Einwirkung von KSCN als auch von $\mathrm{NH}_{4} \mathrm{SCN}$ gleich prompte und zuverlässige Ergebnisse. Auch bei Verwendung der Sulfate erhalten wir eine gute reversible Granulation der Körner, wenn wir die Zellen nach der Behandlung im Farbstoff-Salz Gemisch etwa 1 Stunde lang in reiner $\mathrm{SO}_{4}$-Lösung baden. Dieses Zwischenbad einzuschalten ist deshalb notwendig, weil die Kerne ansonsten stäbchenförmig strukturiert sind (Fig. 2).

Sehr schwierig ist es, körnige Entmischung der Kernkolloide zu erhalten, wenn dem Methylviolett irgendein Nitrat beigemischt 
wird. In diesem Falle muß nach der Färbung bei gleichzeitiger Plasmolyse ein mindestens 24 Stunden dauerndes $\mathrm{Zwischenbad} \mathrm{in}$ reiner Salzlösung $\left(-\mathrm{NO}_{3}\right.$ oder $\left.-\mathrm{Cl}\right)$ eingeschaltet werden, um bei Anwendung des KSCN reversible körnige Entmischung des diffus gefärbten Kernes zu erzielen. Wir können jedoch, um solche Entmischung rascher $\mathrm{zu}$ erhalten, die plasmolysierten Zellen mit diffus gefärbtem Kerne in etwa 2 mol $\mathrm{KNO}_{3}$ stärker plasmolysieren. Dann wird bei Verwendung des KSCN ziemlich prompt die körnige Entmischung erzielt. Das zeigt, daß zur Erzielung der reversiblen Entmischung der Kernkolloide das gesamte System in ein bestimtes labiles Gleichgewichts-

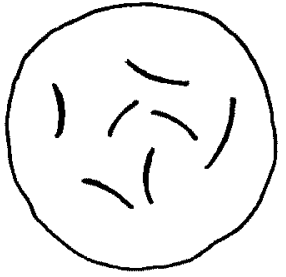

a

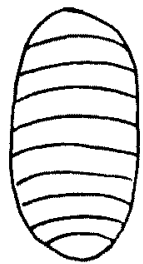

b

Fig. 2. Stäbchenförmige Entmischung des vorher vital diffusgefärbten Zellkernes. Die Stäbchen sind gefärbt, die Zwischensubstanz ist farblos. Die Bedingungen unter welchen die Form a und unter welchen die Form b erhalten werden kann, kann vorläufig nicht angegeben werden. system gebracht werden muß, ein Gleichgewichtssystem, in das die Entmischungssalze leicht eingreifen, es stören und ein neues Gleichgewichtssystem bereiten können. Da außerdem die stärkere Plasmolyse in der 2 mol Lösung der Bereiter einer solchen notwendigen Labilität ist, ließ sich aus der Ordnung in der HoFFMEISTERschen Reihe erwarten, daß das $\mathrm{NH}_{4} \mathrm{SCN}$, in seiner Wirkung stärker als das $\mathrm{KSCN}$, die Entmischung der Kernkolloide, die mit $\mathrm{NO}_{3}$-Farbge-

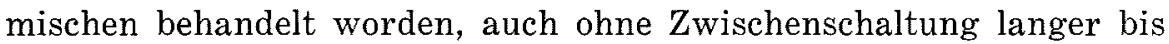
24 Stunden dauernder Wartezeiten oder stärkere Plasmolyse, direkt hervorrufen werde. Die Erwartung wurde bestätigt: bei Verwendung des $\mathrm{NH}_{4} \mathrm{SCN}$ werden die mit Nitrat-Farbstoffgemischen behandelten Kerne sofort, wie in Fig. 2 angegeben, entmischt.

Weil die Formen der Entmischungsbildungen labil sind-körnig bei Verwendung des $\mathrm{KSCN}$, stäbchenförmig bei Benützung des $\mathrm{NH}_{4} \mathrm{SCN}$ - und weil sie in ungefähr gleicher Gestaltung bei der STRUGGERschen Behandlungsmethode erzielt werden, können wir auf ihren Karyotinanteil rückschließen.

Zur Stützung dieses zweiten Wahrscheinlichkeitsbeweises über die Verwandschaft der Entmischungskörner mit dem Karyotin kann ich noch zwei direkte Beobachtungen anführen:

(a) Zellkerne der Allium-Epidermiszellen wurden mit $0.1 \%$ Eosin (5cc) $+0.75 \%$ Essigsäure ( 5 Tropfen) gefärbt und dann in $0.1 \%$ Methylenblau $+\mathrm{Na}_{2} \mathrm{SO}_{4}$ (in Spuren) übertragen. Nach einiger Zeit zeigt der vorhin homogene, diffus gefärbte Kern ein farbloses Kernretikulum (Karyotin) mit gefärbter Karyolymphe. Diese Zellen 
übertragen wir in $1 \mathrm{~mol} \mathrm{KCl}$. Nach einiger Zeit verfeinert sich das Retikulum, die Nukleolen treten stark hervor. Schließlich zerfällt das Retikulum zu Körnern, diese verschwinden und der Kern wird homogen. Bei eingeschalteter Deplasmolyse erscheint das Kernretikulum wieder.-[Konnte nicht wiederholt werden da in anderen Fällen das Kernretikulum bei Plasmolyse nicht zu Körnern zerfällt sondern sich vergröbert, wobei der Kern gänzlich gelifiziert, bei gleichzeitigem Anstieg des Brechungsindex.]

(b) Kerne der Allium-Epidermiszellen wurden in Prune pure $0.1 \%+2 \mathrm{~mol} \mathrm{KCl}$ gefärbt (diffus). Nach Auswaschen in reiner 2 mol KCl-Lösung werden in das Gemisch $0.1 \%$ Methylenbau $+2 \mathrm{~mol}$ $\mathrm{KCl}(1: 1)$ übertragen. Nach 24 Stunden ist der Kern entfärbt. Die Zellen werden in $2 \mathrm{~mol} \mathrm{KCl}(3 \mathrm{ccm})+0.75 \%$ Essigsäure (3 Tropfen) übertragen. Die Kernkolloide werden stäbchenförmig entmischt, die Stäbchen zerfallen nach einiger Zeit zu Körnern.

(D) Genauso wie sich nicht alle Salze gleichmäßig gut zur durchzuführenden reversiblen Entmischung des in gleichzeitig plasmolysierten Zellen vitalgefärbten Kernes eignen, eignen sich auch nicht alle Farbstoffe dazu. Besonders bemerkenswert ist das Verhalten der 3 sehr verwandten Farbstoffe Kristallviolett, Gentianaviolett und Methylviolett zusammen mit $1 \mathrm{~mol} \mathrm{KCl}$ als plasmolysierendes Beimischungssalz. Mit Kristallviolett gefärbte Kerne können nur mit $\mathrm{NH}_{t} \mathrm{SCN}$ entmischt werden, wobei nur ganz wenige Körner entstehen; KSCN ist ganz wirkungslos. Gentianaviolett-Kerne können sowohl mit $\mathrm{NH}_{4} \mathrm{SCN}$ als auch mit $\mathrm{KSCN}$ entmischt werden, dabei sehr leicht mit $\mathrm{NH}_{4} \mathrm{SCN}$, während $\mathrm{KSCN}$ eine nur geringe Menge von Entmischungskörnern gibt. Methylviolett-Kerne entmischen sich gleich gut mit beiden Entmischungssalzen. Eine fundierte Erklärung für das verschiedene Verhalten dieser 3 Farbstoffe kann zur Zeit nicht gegeben werden. Prune pure mit $\mathrm{KCl}$ vermischt eignet sich zu unseren Entmischungsversuchen überhaupt nicht.

Aus der Arbeit Struggers (1933) geht hervor, daß (1) die sauren Farbstoffe die Karyolymphe, die basischen das Karyotin färben und (2) daß die sauren Farbstoffe im sauren Gebiet (I E P $=3,83$ 4,04 bzw. 4,40-4,49) aus der Karyolymphe in das Karyotin wandern. In unserem Falle, da durch die Plasmolyse der basische Farbstoff nicht im Karyotin sondern in der Karyolymphe gespeichert wird, war es jedenfalls interessant zu prüfen, ob die Entmischung durch Änderung der elektrischen Ladung beeinflußt wird. Dazu habe ich mich zweier Methoden bedient (a) der Ansäuerung des Mediums durch $0.5 \%$ Essigsäure, (b) durch Ausnützung der elektrischen Gruppenregel, des sogenannten Kalium-Natriumkontrastes (KELLER). Bekanntlich gehören zur Kaliumgruppe, d.i. zu jenen Salzen die aus 
der Froschhaut auswandern, die also biologisch negativ sind (in Auswahl) :

Basenfarbstoffe sehr stark verdünnt $>\mathrm{NH}_{4}-$ Salze $>$ Kalisalze $>$ Magnesiumsalze in starker Verdünnung, Sulfate und Phosphate.

Zur Natriumgruppe, d.i. zu jenen Salzen, die in der Froschhaut von außen nach innen wandern d.h. biologisch positiv sind, gehören: Wasser, Säurefarbstoffe besonders in angesäuerter Lösung, Lithiumsalze $>$ Natronsalze $>$ Rhodanide $>$ Chloride $>$ Bromide.

Da die Entmischung nach unserer Methode besonders gut gelingt, wenn wir Chloride als Beimischung zum Methylviolett verwenden, und da sie durch Rhodanide, ebenso durch Bromide erzielt werden kann, schien es, als sollte der Natrium-Kaliumkontrast bei der Hervorrufung der beschriebenen Erscheinungen eine besondere Rolle spielen, wenn man annimt, daß gleichsinnige Wirkung der einzelnen Behandlungskomponenten günstig ist zur Erzielung des angestrebten Resultates. Allerdings steigen schon bei bloßer Betrachtung des schon beschriebenen Versuchsergebnisses verschiedene Bedenken auf: denn (1) wirken nicht nur Chloride, die in die Natriumgruppe gehören, entmischungsfördernd, ebenso wirken auch die in die Kaliumgruppe gehörigen Sulfate; (2) es widerspricht auch der theoretischen Erwartung, daß das $\mathrm{NH}_{4} \mathrm{SCN}$ stärker wirkt als das KSCN obwohl jenes der Ordnung in der Gruppe nach negativer ist als dieses. Doch könnte diese Mißstimmung auch so erklärt werden, daß die Form der Entmischungsbildungen von der sonst gewohnten, körnigen abweicht; bekanntlich kommt es in beiden eben angeführten Fällen wenigstens vorübergehend zu Stäbchenbildung.

Wir können uns von der Giltigkeit des Kalium-Natriumkontrastes auf verschiedene Weise überzeugen. Ich habe dazu zwei Möglichkeiten herausgegriffen: (1) bei Behandlung der Zellen mit $\mathrm{KNO}_{3}-$ u. $\mathrm{NaNO}_{3}-$ Methylviolettgemischen konnte bei Anwendung des Entmischungssalzes erwartet werden, daß in den $\mathrm{NaNO}_{3}$-Kernen die Entmischung früher eintritt als in den $\mathrm{KNO}_{3}$-Kernen. Die Beobachtung lehrt, daß diese Erwartung nicht eintritt. (2) Narkose senkt die Potentialdifferenzen (KELLER 1936). Daher dürfte Zugabe von Narkoticis zum Entmischungssalz die Entmischung verzögern. Zugabe von $2 \%$ Äther zu KSCN zeigt im Eintritt der Entmischung keine zeitliche Differenz gegenüber der Wirkung reinen KSCN.

Wir kommen zum Schluß, daß am Zustandekommen der Entmischung im mit Methylviolett diffus gefärbten Kerne der plasmolysierten Zelle die elektrostatischen Ladungen keinen primären Anteil besitzen dürften. Dafür zeugt auch der Umstand, daß auch durch das KSCN keine Änderung des Resultates festzustellen war.

Eigentlich überrascht diese Tatsache nicht sonderlich, wenn wir 
z.B. an den Mechanismus der Vitalfärbung des Kernes zurückdenken. Da ist es, mindestens bei Färbung mit Eosin, so (BANK) daß der bis dahin unsichtbare Zellkern sich zuerst optisch differenziert, bevor er den Farbstoff zu speichern beginnt (siehe auch KAMNEW 1934 für Neutralrot). Es verändert sich also zuerst das Gleichgewicht der Zelle, damit vielleicht zusammen die einzelnen elektrostatischen Ladungen, worauf erst der Farbstoff gespeichert wird. Ich erachte es daher nicht für ganz genau, den Zellkern allgemein als anodisch zu bezeichnen, sondern ich möchte vielmehr einengen, daß der optisch differenzierte Zellkern anodisch ist. Über seine elektrischen Eigenschaften im optisch nicht differenzierten Zustand können wir vorläufig schwerlich etwas Positives aussagen.

In Erwägung aller berichteten Tatsachen können wir die vorläufig wohl am wenigsten gewaltsame Schlu folgerung ziehen, daß die körnige Entmischung der Kernkolloide nicht nur physikalisch sondern mindestens zu großen Teile chemisch bedingt ist. Der genaue Mechanismus der zur Entmischung führt bleibt unbekannt.

\section{Zusammenfassung}

1. Die Kernkolloide der Epidermiszellen von Allium cepa im Gemisch $1 \mathrm{~mol} \mathrm{KCl}+0.1 \%$ Methylviolett $(1: 1)$ vital diffus gefärbt, können durch KSCN körnig und reversibel entmischt werden (Fig. 1).

2. Die Körner können einigemal (bis zu 3 mal) erzeugt und wieder zum Verschwinden gebracht werden.

3. Als Beimischungssalz zum Methylviolett eignen sich auch andere Chloride: $\mathrm{NaCl}, \mathrm{LiCl}, \mathrm{NH}_{4} \mathrm{Cl}$; schwieriger findet die Entmischung statt, bei Verwendung von Sulfaten $\left(\mathrm{Na}_{2} \mathrm{SO}_{4}, / \mathrm{NH}_{4} / 2 \mathrm{SO}_{4}\right)$, und sehr schwer gestaltet sich die Entmischung, wenn Nitrate $\left(\mathrm{KNO}_{3}\right.$, $\mathrm{NaNO}_{3}, \mathrm{NH}_{4} \mathrm{NO}_{3}, \mathrm{Ca} / \mathrm{NO}_{3} / 2$ ) dem Farbstoff beigemischt werden.

4. Die Entmischung nach der Nitratbehandlung kann leichter gemacht werden (a) durch vorhergehende verstärkte Plasmolyse ( 2 mol Lösungen) der Zellen oder (b) durch Verwendung von $\mathrm{NH}_{4}$. SCN als Entmischungssalz. In diesem Falle kommt es jedoch nicht zu körniger sondern zu stäbchenförmiger Entmischung (Fig. 2).

5. Auch eignen sich nicht alle Farbstoffe zur Hervorrufung der körnigen Entmischung. Prune pure ist gänzlich untauglich. Kristallviolett gibt mit $\mathrm{KCl}$ als Beimischung schwache körnige, rever.. sible Entmichung nur bei Anwendung von $\mathrm{NH}_{4} \mathrm{SCN}$ als Entmischungssalz, mit KSCN gibt es keine Entmischung; Gentianaviolett mit $\mathrm{KCl}$ gibt mit $\mathrm{KSCN}$ schwache, mit $\mathrm{NH}_{4} \mathrm{SCN}$ gute Entmischung, Methylviolett gibt sowohl mit $\mathrm{KSCN}$ als auch mit $\mathrm{NH}_{4} \mathrm{SCN}$ gute Entmischung. 
6. Beim Zustandekommen der Entmischung gilt der KaliumNatriumkontrast nicht. Es wird die Annahme ausgesprochen, daß chemische Affinitäten neben den physikalischen Eigenschaften eine sehr beachtenswerte Rolle spielen dürften.

7. Es wird der Beweis dafür zu führen gesucht, daß bei vitaler Diffusfärbung des Kernes mit basischen Farbstoffen-bei gleichzeitiger Plasmolyse-diese Färbungsart nicht durch Änderung des Chromatinanteiles des Kernes, sondern durch Veränderung des phasischen Gleichgewichtes im Kerne bedingt wird.

\section{Schrifttum}

Albach W., Protoplasma 5, 1929; 412.

Bank O., Protoplasma 18, 1933 a ; 620 .

- Protoplasma 19, $1933 \mathrm{~b} ; 125$.

- $\quad$ Protoplasma 25, 1936 a ; 188.

- Verhandlungen d. Naturforsch. Ver. Brün 67, 1936 b; 107.

Becker W. A. u. Beckerowa Z., Acta Soc. Bot. Poloniae 11, 1934 ; 367.

Bertalanffy L. V., Theoretische Biologie I. Borntraeger Berlin 1932.

Gicklhorn J., Protoplasma 2, 1927; 1 .

Keller R., Der elektrische Faktor der Ernährung. S. Karger-Verlag, Berlin 1936.

Martens P., Cellule, 38, $1927 ; 67$.

Sharp L. W., Bot. Gazette 88, $1929 ; 249$.

Strugger S., Protoplasma 10, 1930;363.

- Planta 18, $1933 ; 561$.

Teleżyński H., Acta Soc. Bot. Poloniae 7, 1930 ; 381. 